EESTI NSV TEADUSTE AKADEEMIA TOIMETISED. X KÖIDE

FUOSIKALIS-MATEMAATILISTE JA TEHNILISTE TEADUSTE SEERIA. 1961, NR. 1

ИЗВЕСТИЯ АКАДЕМИИ НАУК ЭСТОНСКОЙ ССР. ТОМ Х

СЕРИЯ ФИЗИКО-МАТЕМАТИЧЕСКИХ И ТЕХНИЧЕСКИХ НАУК, 1961, № 1

\title{
ЛИНЕЙЫЕ ПРЕОБРАЗОВАНИЯ НЕКОТОРЫХ КЛАССОВ ДВОЙНЫХ ПОСЛЕДОВАТЕЛЬНОСТЕЙ
}

\author{
и. кулль,
}

\section{кандидат физико-математических наук}

Целью настоящей статьи является разработка метода нахождения необходимых и достаточных условий для линейных преобразований некоторых классов двойных последовательностей. В статье приводятся также два примера применения полученных результатов для исследования сходимости произведения двух рядов.

§ 1. В статье рассматриваются двойные последовательности $x=$ $=\left\{\xi_{k l}\right\}$ с комплексными членами. Рассмотрению подвергаются следующие классы двойных последовательностей: $\mathrm{m}, \mathrm{mc}, \mathrm{r}, \mathrm{l}, \mathbf{c}_{\lambda}, \mathrm{c}_{\mathrm{y}}^{\mathrm{m}}$ и $\mathrm{m}_{\gamma}^{\mathrm{m}}$, определения которых можно найти в статье [5] (стр. 5-6) *.

Кроме того, используются и другие классы двойных последовательностей, определения которых даны ниже. Пусть задана двойная последовательность $\left\{\Phi_{k l}\right\}$, удовлетворяющая условию $\Phi_{k l}>0(k, l=0,1, \ldots)$. При помощи ее определяем классы двойных последовательностей $\mathrm{m}_{\Phi}$, $\mathrm{mc}_{\Phi}, \mathbf{r}_{\Phi}$ и $\mathbf{l}_{\Phi}$ следующим образом: считаем, что $x=\left\{\xi_{k l}\right\} \in \alpha_{\Phi}$, если $\left\{\frac{\xi_{k l}}{\Phi_{k l}}\right\} \in \alpha(\alpha=\mathrm{m}, \mathrm{mc}, \mathrm{r}$ или 1$)$. В частном случае, если $\Phi_{k l}=1$, получаем классы $\mathbf{m}, \mathrm{mc}, \mathrm{r}$ и 1.

Пусть задано счетное множество двойных последовательностей $\Phi^{(p)}=\left\{\Phi_{k l}^{(p)}\right\} \quad(p=0,1, \ldots)$, удовлетворяющих условию $\Phi_{k l}^{(p)}>0 \quad(k, l$, $p=0,1, \ldots)$. При помоши этих последовательностей можем определить классы $\mathbf{m}_{\Phi,}^{(p)}, \mathbf{m c}_{\Phi}^{(p)}, \mathbf{r}_{\Phi}^{(p)}$ и $\mathbf{1}_{\Phi}^{(p)}$, где классы $\alpha_{\Phi}^{(p)}(\alpha=\mathbf{m}, \mathbf{m c}, \mathbf{r}$ или 1) определены при помощи $\Phi(p)$. При помощи этих классов получаем новые классы $\mathbf{m}_{\Phi}^{*}=\bigcup_{p} \mathbf{m}_{\Phi}^{(p)}, \mathbf{m c}_{\Phi}^{*}=\bigcup_{p} \mathbf{m c}_{\Phi}^{(p)}, \mathbf{r}_{\Phi}^{*}=\bigcup_{p} \mathbf{r}_{\Phi}^{(p)}$ и $\mathbf{1}_{\Phi}^{*}=\bigcup_{p} \mathbf{l}_{\Phi}^{(p)}$.

В частности, положив

$$
\Phi_{k l}^{(p)}=\left\{\begin{array}{l}
1, \text { если } k, l>p, \\
\chi(k, l), \text { в остальных случаях, }
\end{array}\right.
$$

получаем $\mathrm{m}_{\chi}^{\mathrm{m}}=\mathrm{m}_{\Phi}^{*}$ и $\mathrm{c}_{\chi}^{\mathrm{m}}=\mathrm{mc}_{\mathrm{q}}^{*}$.

- Кроме этих классов можно рассматривать и другие классы ограниченных двойных последовательностей, как $B C N, R C N, R C R N$ и т. д., которые определены в работе Гамильтона [']. Все последующие математические конструкции в той же мере возможны и для этих классов (и не только для двойных последовательностей). В настоящей статье выбраны, по нашему мнению, наиболее существенные классы двойных последовательностей. 
§ 2. Пусть $\alpha$ и $\beta$ некоторые классы двойных последовательностей. Рассматриваем матричное преобразование

$$
\dot{\xi}_{m n}^{\prime}=\sum_{k, l} a_{m n k l} \xi_{k l} \quad(m, n=0,1, \ldots),
$$

где $a_{m n k l}(m, n, k, l=0,1, \ldots)$ - комплексные числа. Ищем необходимые и достаточные условия для того, чтобы преобразование (1) переводило все последовательности $x=\left\{\xi_{k l}\right\}$ класса $\alpha$ в последовательности $x^{\prime}=\left\{\xi_{m n}^{\prime}\right\}$ класса $\beta$. При этом подразумевается, что двойные ряды в (1) сходятся при всех $x \in \alpha$ и $m, n=0,1, \ldots$ Искомые необходимые и достаточные условия назовем необходимыми и достаточными (или коротко: точными) условиями линейного преобразования $\alpha \rightarrow \beta$.

Поставленную проблему нахождения точных условий можно peшить методами функционального анализа, как это сделано в статьях $\left.{ }^{2}\right]$ и [5]. Для нахождения точных условий линейных преобразований, рассматриваемых в настоящей статье, можно обойтись без прямого применения методов функционального анализа, сведя их нахождение к применению точных условий уже известных линейных преобразований.

В первую очередь изучим линейные преобразования $\alpha_{\Phi} \rightarrow \beta_{\psi}$, где $\alpha_{\Phi}$ - один из классов $\mathrm{m}_{\Phi}, \mathrm{mc}_{\Phi}, \mathbf{r}_{\Phi}$ или $\mathbf{1}_{\Phi}$ и $\beta_{\psi}-$ один из классов $\mathbf{m}_{\psi}$, $\mathbf{m c}_{\psi}, \mathbf{r}_{\psi}$ или $\mathbf{1}_{\psi}$. В этом случае $\left\{\frac{\xi_{k l}}{\Phi_{k l}}\right\}=\left\{\zeta_{k l}\right\} \in \alpha$ и $\left\{\frac{\xi_{m n}}{\psi_{m n}}\right\}=\left\{\eta_{m n}\right\} \in \beta$. Члены последовательностей $x$ и $x^{\prime}$ можно привести к виду

и

$$
\xi_{k l}=\Phi_{k l} \zeta_{k l} \quad(k, l=0,1, \ldots)
$$

$$
\xi_{m n}^{\prime}=\psi_{m n} \eta_{m n} \quad(m, n=0,1, \ldots),
$$

вследствие чего равенство (1) перепишется так:

$$
\eta_{m n}=\sum_{k, l} \frac{\Phi_{k l} a_{m n k l}}{\psi_{m n}} \zeta_{k l} \quad(m, n=0,1, \ldots)
$$

где $\left\{\zeta_{k l}\right\} \in \alpha$ и $\left\{\eta_{m n}\right\} \in \beta$. Очевидно, что точные условия линейного преобразования $\alpha_{\Phi} \rightarrow \beta_{\psi}$ для матрицы $A=\left(a_{m n k l}\right)$ совпадают с точными условиями линейного преобразования $\alpha \rightarrow \beta$ для матрицы

$$
B=\left(\frac{\Phi_{k l} a_{m n k l}}{\psi_{m n}}\right)
$$

Последние найдены в работах $\left.\left.{ }^{1}\right],{ }^{2}\right]$ и [5], за исключением точного условия для линейных преобраозваний $\alpha \rightarrow 1(\alpha \neq 1)$, которое найдем в $\S 3$ настоящей статьи.

Далее рассматриваем линейные преобразования $\alpha_{\Phi}^{*} \rightarrow \beta_{\psi}$, где $a_{\Phi}^{*}$ - один из классов $\mathrm{m}_{\Phi}^{*}, \mathrm{mc}_{\Phi}^{*}, \mathbf{r}_{\Phi}^{*}$ или $\mathbf{1}_{\Phi}^{*}$ и $\beta_{\psi}$ имеет то же самое значение, что и выше. Для того, чтобы матрица $A=\left(a_{m n k l}\right)$ удовлетворяла точным условиям линейного преобразования $\alpha_{\Phi}^{*} \rightarrow \beta_{\psi}$, она должна удовлетворять точным условиям линейного преобразования $\alpha_{\Phi}^{(p)} \rightarrow \beta_{\psi}$ для любого $p=0,1, \ldots$. Следовательно, матрицы

$$
B_{p}=\left(\frac{\Phi_{k l}^{(p)} a_{m n k l}}{\psi_{m n}}\right)
$$


должны удовлетворять точным условиям линейного преобразования $\alpha \rightarrow \beta$ для любого $p=0,1, \ldots$.

Наконец, рассмотрим линейные преобразования $\alpha_{\Phi}^{*} \rightarrow \mathbf{c}_{\lambda}$. Аналогично рассуждая, придем к заключению, что в данном случае матрицы

$$
C_{p}=\left(\Phi_{k l}^{(p)} a_{m n k l}\right)
$$

должны удовлетворять точным условиям линейного преобразования $\alpha \rightarrow \mathbf{c}_{\lambda}$ для любого $p=0,1, \ldots$ Соответствующие условия даны в [5] (за исключением случая $\mathbf{m} \rightarrow \mathbf{c}_{\lambda}$, который приведем ниже).

Приведем теперь два примера получения точных условий линейных преобразований.

Пример 1. Найдем точные условия $\mathrm{mc}_{\Phi}^{*} \rightarrow \mathrm{mc}_{\psi}$. Матрицы $B_{p}$ должны для любого $p=0,1, \ldots$ удовлетворять условиям $\mathrm{mc} \rightarrow \mathrm{mc}$, т. е. условиям $c_{1}, d_{3}$ и $d_{4}$ (см. [5], стр. 19-21). Применяя их к матрицам $\dot{B}_{p}$, получаем:

$$
\begin{array}{lll}
1^{\circ} & \sum_{k, l} \Phi_{k l}^{(p)}\left|a_{m n k l}\right| \leqslant M_{p} \psi_{m n} & (m, n, p=0,1, \ldots), \\
2^{\circ} & \lim _{m, n \rightarrow \infty} \frac{1}{\psi_{m n}} \sum_{k, l} \Phi_{k l}^{(p)} a_{m n k l}=a_{p} & (p=0,1, \ldots), \\
3^{\circ} & \lim _{m, n \rightarrow \infty} \sum_{k} \Phi_{k l}^{(p)}\left|\frac{a_{m n k l}}{\psi_{m n}}-b_{k l}\right|=0 & (l, p=0,1, \ldots), \\
& \lim _{m, n \rightarrow \infty} \sum_{l} \Phi_{k l}^{(p)}\left|\frac{a_{m n k l}}{\psi_{m n}}-b_{k l}\right|=0 & (k, p=0,1, \ldots),
\end{array}
$$

где $b_{k l}=\lim _{m, n \rightarrow \infty} \frac{a_{m n k l}}{\psi_{m n}} \quad(k, l=0,1, \ldots)$.

Условия $1^{\circ}, 2^{\circ}$ и $3^{\circ}$ и являются искомыми точными условиями $\mathbf{m c}_{\phi}^{*} \rightarrow \mathbf{m c}_{\psi}$. В частности, из этих условий можно вывести точные условия для $\mathrm{mc}_{\Phi}^{*} \rightarrow \mathrm{mc}, \mathrm{mc}_{\Phi} \rightarrow \mathrm{mc}_{\psi}, \mathrm{mc}_{\Phi} \rightarrow \mathrm{mc}, \mathrm{mc} \rightarrow \mathrm{mc}_{\psi}$ и $\mathrm{mc} \rightarrow \mathrm{mc}$.

Пр имер 2. Найдем точные условия $\mathrm{m}_{\Phi}^{*} \rightarrow \mathbf{c}_{\lambda}$. Матрицы $C_{p}$ должны для любого $p=0,1, \ldots$ удовлетворять условиям $\mathbf{m} \rightarrow \mathbf{c}_{\lambda}$. Эти условия легко найти, применяя использованный в статье [5] метод функционального анализа. Учитывая существование преобразования (1), получаем условие $a_{1}$ :

$$
\sum_{k, l}\left|a_{m n k l}\right|<+\infty \quad(m, n=0,1, \ldots),
$$

учитывая ограниченность в смысле $\mathbf{m} \lambda$, получаем условие $\overline{c_{1}}$ :

$$
\begin{array}{ll}
\sum_{k, l}\left|a_{m n k l}\right| \leqslant M(\lambda) & \text { (при любых } \lambda \geqslant 1-\text { и для всех } m, n, \text { удовлетворяющих } \\
& \text { условию } \left.\frac{1}{\lambda} \leqslant \frac{m}{n} \leqslant \lambda\right) .
\end{array}
$$

Учитывая сходимость в смысле $c_{\lambda}$ на основном множестве пространства m, получаем

$$
\lim _{(m, n) \lambda \rightarrow \infty} \sum_{k, l \in G} a_{m n k l}=a(G),
$$

где $G$ означает любое подмножество множества пар неотрицательных 
целых чисел. ${ }^{\star}$ Последнее условие можно привести к следующему более удобному виду:

$$
\lim _{(m, n)} \sum_{\lambda \rightarrow \infty}\left|a_{m n k l}-a_{k l}\right|=0,
$$

где $a_{k l}=\lim _{(m, n)_{\lambda} \rightarrow \infty} a_{m n k l} \quad(k, l=0, \ldots) .^{* *}$

Точные условия линейного преобразования $\mathbf{m}_{\Phi}^{*} \rightarrow \mathbf{c}_{\lambda}$, на основе сказанного, суть следующие:

$$
\begin{aligned}
& 1^{\circ} \sum_{k, l} \Phi_{k l}^{(p)}\left|a_{m n k l}\right|<+\infty \quad(m, n, p=0,1, \ldots), \\
& 2^{\circ} \quad \sum_{k, l} \Phi_{k l}^{(p)}\left|a_{m n k l}\right| \leqslant M_{p}(\lambda) \\
& (p=0,1, \ldots, \text { при любых } \lambda \geqslant 1 \text { и } \\
& \text { для всех } m, n, \text { удовлетворяюших } \\
& \text { условию } \left.\frac{1}{\lambda} \leqslant \frac{m}{n} \leqslant \lambda\right) \text {, } \\
& 3^{\circ} \lim _{(m, n)_{\lambda} \rightarrow \infty} \sum_{k, l} \Phi_{k l}^{(p)}\left|a_{m n k l}-a_{k l}\right|=0 \quad(p=0,1, \ldots) \text {, }
\end{aligned}
$$

где $a_{k l}=\lim _{(m, n)_{\lambda} \rightarrow \infty} a_{m n k l}$.

В частности, из этих условий можно вывести точные условия для $\mathbf{m}_{\Phi} \rightarrow \mathbf{c}_{\lambda}$ и $\mathbf{m} \rightarrow \mathbf{c}_{\lambda}$.

§ 3. Теперь выведем точное условие линейных преобразований $\alpha \rightarrow 1$ ( $\alpha=\mathrm{m}$, mс или $\mathrm{r})$. Вывод этого условия аналогичен выводу соответственного условия в случае простых последовательностей. ${ }^{\star \star \star}$

Нам нужна следующая

Л емма. Для того, чтобы $x \in 1$, необходимо $и$ достаточно, чтобы сущцествовала постоянная $K$, такая что

$$
\left|\sum_{k, l \in M} \xi_{k l}\right| \leqslant K
$$

для любого $\mathfrak{M}$, где $\mathfrak{M}$ означает конечное множество пар неотрицательных целых чисел.

Д оказ ательство. Необходимость условия (3) очевидна. Докажем достаточность. Поскольку $\xi_{k l}=c_{k l}+i d_{k l} \quad(k, l=0,1, \ldots)$, то

$$
\left|\sum_{k, l \in \mathfrak{M l}} c_{k l}\right| \leqslant\left|\sum_{k, l \in \mathfrak{M} l} \xi_{k l}\right| \leqslant K
$$

имеет место для любого $\mathfrak{M}$. Выбрав $\mathfrak{M}$ таким, что $c_{k l} \geqslant 0$, получаем

* Основное множество пространства m составляется всевозможными последовательностями, членами которых являются числа 0 или 1 в произвольном порядке (см. [ $\left.{ }^{2}\right]$, стр. 158-159).

* См. условие $\left(d_{5}\right)$ в работе Гамильтона ['].

*** См. Пейернмхофф [3], стр. 141-142. 


$$
\sum_{k, l_{e} \mathfrak{M}}\left|c_{k l}\right| \leqslant K
$$

вследствие чего неотрицательные вещественные части комплексных чисел $\xi_{k l}$ образуют абсолютно сходящийся ряд. Таким же образом находим, что все отрицательные вещественные, неотрицательные мнимые и отрицательные мнимые части комплексных чисел $\xi_{k l}$ образуют абсолютно сходящиеся ряды. Неравенство (4) показывает, что сумма ни одного из этих четырех рядов не превосходит $K$. Следовательно,

$$
\sum_{k, l}\left|\xi_{k l}\right| \leqslant 4 K,
$$

чем лемма доказана.

Т ео рем а. Для того, чтобы преобразование (1) переводило все последовательности класса $\alpha(\alpha=\mathbf{m}, \mathbf{m c}$ или r) в последовательности класса 1 , необходимо и достаточно существование постоянной $M$, такой чTO

$$
\left|\sum_{m, n \in M i} \sum_{k, l \in S i} a_{m n k l}\right| \leqslant M
$$

для любых $\mathfrak{M}$ и $\Re$, где $\mathfrak{M}$ и $\Re$ - конечные множества пар неотрицательных чүелых чисел.

Доказ а тельство. Для сходимости ряда (1) при любом $x \in a$ и $m, n=0,1, \ldots$ необходимо и достаточно условие $a_{1}{ }^{\star}{ }^{\circ}$ Для того, чтобы $x^{\prime} \in 1$, по лемме необходимо и достаточно условие (3), которое в данном случае принимает вид

$$
\left|\sum_{m, n \in \mathfrak{M}}\left(\sum_{k, l} a_{m n k l \xi k l}\right)\right| \leqslant K,
$$

где $K$ не зависит от $\mathfrak{M}$, но может зависеть от $\left\{\xi_{k l}\right\}$. Так как ряды в условии (7) при любом $m, n=0,1, \ldots$ сходятся и $\mathfrak{M}-$ конечное множество, можно условие (7) представить в виде

$$
\left|\sum_{k, l}\left(\sum_{m, n \in \mathfrak{M} l} a_{m n k l}\right) \xi_{k l}\right| \leqslant K .
$$

Поскольку все конечные подмножества $\mathfrak{M}$ пар неотрицательных целых чисел можно упорядочить в последовательность, то выражение в скобках в условии (8) можно рассматривать как линейное преобразо вание $\alpha \rightarrow \mathrm{m}$, точным условием которого является $c_{1}$, что в данном слу-
чае дает

$$
\sum_{k, l}\left|\sum_{m, n \in \mathfrak{M l}} a_{m n k l}\right| \leqslant M \quad \text { (для любого } \mathfrak{M} \text { ). }
$$

Согласно условию (3) леммы можем неравенство (9) переписать в виде (6), чем теорема доказана.

Относительно этой теоремы сделаем некоторые примечания.

-. См. Гамильтон ['], стр. 41 и 47.

- См. там же, стр. 42 н 47.

2 ENSV TA Toimetised T-1 61 
При ме ч а н е 1. Условия (6), (9) и

$$
\sum_{m, n}\left|\sum_{k, l \in \Omega} a_{m n k l}\right| \leqslant M^{\prime}
$$

равносильны. Равносильность их вытекает из доказанной выше леммы Условие $a_{1}$ существования преобразования (1) содержится в самих условиях (6), (9) и (10).

При меча н ие 2. Условие $\sum_{m, n} \sum_{k, l}\left|a_{m n k l}\right|<+\infty$ достаточно для преобразования $\alpha \rightarrow 1$ ( $\alpha=\mathbf{m}, \mathbf{m c}$ или $\mathbf{r})$, но не необходимо. Чтобы опровергнуть ее необходимость применим аналогичный результат для простых рядов. А именно, Пейеримхофф показал, что матрица с элементами

$$
a_{m k}=\frac{1}{m+1} \int_{\ln (k+1)}^{\ln (k+2)} e^{i m t} \frac{d t}{(t+1)^{2}}
$$

переводит все ограниченные простые последовательности в абсолютно сходящиеся, в то время как

$$
\sum_{m} \sum_{k}\left|a_{m k}\right|=\infty *^{*}
$$

Положив в (1)

$$
a_{m n k l}=\left\{\begin{array}{l}
a_{m k}, \text { если } n=m \text { и } l=k, \\
0, \text { в остальных случаях, }
\end{array}\right.
$$

находим, что преобразование (1) переводит все последовательности $\left\{\xi_{k l}\right\} \in \mathrm{m}$ в последовательности $\left\{\xi_{m n}^{\prime}\right\} \in 1$, в то время как

$$
\sum_{m, n} \sum_{k, l}\left|a_{m n k l}\right|=\sum_{m} \sum_{k}\left|a_{m k}\right|=\infty .
$$

П ри ме ч а н е 3 . Учитывая то обстоятельство, что результаты Гамильтона [1] справедливы для $q$-кратных $(q \geqslant 1)$ последовательностей, можно лемму и теорему настоящего параграфа без существенных изменений сразу доказать для $q$-кратных последовательностей. При этом $\mathfrak{M}$ и $\Omega$ нужно понимать как конечные множества упорядоченных систем $q$ неотрицательных целых чисел.

Примечание 4. Условие (6) (или равносильные ему условия (9) или (10)) является точным условием линейного преобразования $a \rightarrow 1$ не только в случаях $\alpha=\mathrm{m}, \mathrm{mc}$ или $\mathrm{r}$, но также в случаях $\alpha=B C N$, $R C N, R C R N$ и других классов ограниченных последовательностей, определенных в статье [1]. Это объясняется тем, что точное условие преобразования $\alpha \rightarrow \mathrm{m}$ для всех этих классов $\alpha$ то же самое $-c_{1}$. Применение условия $c_{1}$ к (8) во всех этих случаях дает условие (9).

$\S 4$. Наконец, применим полученные условия для исследования проблемы умножения двойных рядов, где ряд-произведение составляется по правилу Коши. По этому правилу член $w_{s t}$ ряда-произве-

- См. Пейеримхофф [4], стр. 35-36. 
дения $\sum w_{k l}$ двух рядов $\sum u_{k l}$ и $\sum v_{k l}$ определяется следующим образом:

$$
w_{s t}=\sum_{k, l=0}^{s, t} v_{s-k, t-l} u_{k l} \quad(s, t=0,1, \ldots),
$$

где, суммируя по $s$ и $t$, получаем для частичной суммы $W_{m n}$ ряда-произведения следующее выражение:

$$
W_{m n}=\sum_{k, l=0}^{m, n} v_{m-k, n-l} U_{k l} \quad(m, n=0,1, \ldots) .
$$

Ищем необходимые и достаточные условия для того, чтобы из $\left\{U_{k l}\right\} \in \mathbf{r}_{\Phi}$ всегда следовало $\left\{W_{m n}\right\} \in \mathbf{m c}_{\psi}$. Соотношение (12) можно рассматривать как линейное преобразование, которое должно удовлетворять точным условиям $\mathbf{r}_{\Phi} \rightarrow \mathbf{m c}_{\psi}$. Таким образом, для решения поставленной проблемы умножения рядов получаем следующие точные условия:

$$
\begin{aligned}
& 1^{\circ} \sum_{k, l=0}^{m, n} \Phi_{k l}\left|v_{m-k, n-l}\right| \leqslant M \psi_{m n} \quad(m, n=0,1, \ldots), \\
& 2^{\circ} \quad \lim _{m, n \rightarrow \infty} \frac{1}{\psi_{m n}} \sum_{k, l=0}^{m, n} \Phi_{k l} v_{m-k, n-l}=a, \\
& 3^{\circ} \quad \lim _{m, n \rightarrow \infty} \frac{1}{\psi_{m n}} \sum_{k=0}^{m} \Phi_{k l} v_{m-k, n-l}=a_{l} \quad(l=0,1, \ldots) . \\
& \lim _{m, n \rightarrow \infty} \frac{1}{\psi_{m n}} \sum_{l=0}^{n} \Phi_{k l} v_{m-k, n-l}=a_{k}^{\prime} \quad(k=0,1, \ldots) . \\
& \lim _{m, n \rightarrow \infty} \frac{v_{m-k, n-l}}{\psi_{m n}}=a_{k l} \quad(k, l=0,1, \ldots) *^{*}
\end{aligned}
$$

В заключение докажем еще одну теорему об умножении двойных рядов, в которой воспользуемся точным условием для $\alpha \rightarrow 1(\alpha \neq 1)$.

Теорема. Для того, чтобы ряд-произведение $\sum w_{k l}$ двух рядов $\sum u_{k l}$ и $\sum v_{k l}$ было абсолютно сходящимся для любого $\sum u_{k l}$, частичные суммы которого принадлежат классу $\alpha(\alpha=\mathbf{m}, \mathbf{m c}, \mathbf{r}, \ldots, R C R N)$, необходимо и достаточно, чтобы $v_{k l}=0(k, l=0,1, \ldots)$.

Доказательство. Достаточность условия теоремы сразу вытекает из (11). Для доказательства необходимости перепишем (11) в виде

- Условие $2^{\circ}$ дает идею для определения нового метода суммирования, где за обобщенную сумму ряда $\sum u_{k l}$ понимается предел $\lim _{m, n \rightarrow \infty} \frac{1}{b_{m n}} \sum_{k, l=0}^{m, n} a_{m-k, n-l} U_{k l}$. Определенный метод суммирования является обобшением метода Вороного-Нерлунда. 


$$
w_{m n}=\sum_{k, l=0}^{m, n} \Delta_{k l} v_{m-k, n-l} \cdot U_{k l} \quad(m, n=0,1, \ldots),
$$

где $\left\{U_{k l}\right\} \in \alpha$ и $\left\{w_{m n}\right\} \in 1$. Следовательно, матрица $\left(\Delta_{k l} v_{m-k, n-l}\right)$ должна удовлетворять точному условию $\alpha \rightarrow \mathbf{1}$, т. е. должно выполняться

$$
\left|\sum_{m, n \in M} \sum_{k, l \in \Re} \Delta_{k l} v_{m-k, n-l}\right| \leqslant M .
$$

Взяв в неравенстве (14) $\Re\{(0,0)\}$, получаем, согласно лемме параграфа 3, что ряд

$$
\sum_{m, n} \Delta_{m n} v_{m n}
$$

сходится абсолютно.

Теперь покажем, что $v_{00}=0$. Для этого определим множества $\mathfrak{M}_{j}=\Re_{j}=\left\{(0,0),(2,2), \ldots,\left(n_{j}, n_{j}\right)\right\}, \quad$ где $n_{j}=2 n_{j-1}+2 \quad(j=1,2, \ldots)$ и $n_{0}=0$. Вставляя $\mathfrak{M}_{j}$ и $\mathfrak{R}_{j}$ в соотношение (14), получаем

$$
\left|(j+1) v_{00}+\Delta_{22} v_{22}+\ldots+\Delta_{n_{j} n_{j}} v_{n_{j} n_{j}}\right| \leqslant M .
$$

Учитывая абсолютную сходимость ряда (15), должно

$$
\left|(j+1) v_{00}\right| \leqslant M
$$

для любого $j$, откуда следует $v_{00}=0$.

Допустим, что $v_{k l}=0$, если $k \leqslant s-1$ или $l \leqslant t-1$ и докажем, что тогда $v_{s t}=0$. Для доказательства последнего определим множества $\mathfrak{\Re}_{j}$ как выше, но множества $\mathfrak{M}_{j}$ определим следующим образом: если $(p, q) \in \mathfrak{\Omega}_{i}$, то $(p+s, q+t) \in \mathfrak{M}_{j}$. Вставляя эти множества в соотношение (14), получаем неравенство, аналогичное (16), лишь с тем отличием, что все первые индексы больше на $s$ и все вторые индексы больше на $t$. Отсюда следует, что

$$
\left|(j+1) v_{s t}\right| \leqslant M
$$

для любого $j$, откуда, в свою очередь, $v_{s t}=0$. Этим теорема доказана.

Аналогичная теорема имеет место и для простых рядов.

\section{ЛИТ Е Р АТ У РА}

1. H. J. Hamilt o n, Transformations of multiple sequences, Duke Math. J., Vol. 2, No. $1,1936,29-60$.

2. J. D. Hill and H. J. $\mathrm{Hamilt}$ on, Operation theory and multiple sequence transformations, Duke Math. J., Vol, 8, No. 1, 1941, 154-162.

3. A. P e ye rim h of f, Uber Summierbarkeitsfaktoren und verwandte Fragen bei Cesàroverfahren I, Publications de l'Institut Mathématique, VIII, 1955, 139-156.

4. A. Peyerim h of f, Ober ein Lemma von Herrn H. C. Chow, J. London Math. Soc., 32, 1957, 33-36.

5. И. Г. К улль, Умножение суммируемых двойных рядов, Уч. зап. Тартуск. ун-та, $62,1959,3-59$. 


\section{TEATAVATE KAHEKORDSETE JADADE KLASSIDE LINEAARTEISENDUSED}

\section{Kull,}

\section{füüsikalis-matemaatiliste teaduste kandidaat}

\section{Resümee}

Artiklis vaadeldakse kahekordseid jadasid $x=\left\{\xi_{k l}\right\}$ kompleksarvuliste liikmetega. Töös defineeritakse järgmised kahekordsete jadade kiassid: $m_{\Phi}, \quad \mathbf{m c}_{\Phi}, \mathbf{r}_{\Phi}, \mathbf{1}_{\Phi}, \mathbf{m}_{\Phi}^{*}$, $\mathbf{m c}_{\Phi}^{*}, \mathbf{r}_{\Phi}^{*}$ ja $\mathbf{l}_{\Phi}^{*}$, mis on tuntud kahekordsete jadade klasside $\mathbf{m}, \mathbf{m c}, \mathbf{r}$ ja $\mathbf{l}$ üldistuseks $(\$ 1)$.

Töös antakse meetod tarvilike ja piisavate tingimuste saamiseks lineaarteisenduste $\alpha \rightarrow \beta$ jaoks, kus $\alpha$ tähistab mistahes klassi ülaltoodud klassidest, $\beta$ aga on kas klass $\mathrm{m}_{\psi}, \mathbf{m c}_{\psi}, \mathbf{r}_{\psi}, \mathbf{1}_{\psi}$ või $\mathbf{c}_{\lambda}(\S 2)$.

Edasi antakse tarvilik ja piisav tingimus lineaarteisenduste $\alpha \rightarrow \mathrm{I}(\alpha=\mathrm{m}, \mathrm{mc}, \mathrm{r})$ jaoks $(\$ 3)$.

Töös tuuakse kaks näidet saadud tingimuste rakendamise kohta Cauchy korrutisrea koonduvuse uurimiseks $(\$ 4)$.

Tartu Riiklik Olikool

Saabus toimetusse

24. VII 1959

\section{LINEAR TRANSFORMATIONS OF CERTAIN CLASSES OF DOUBLE SEQUENCES}

\section{Kull}

\section{Summary}

In the present paper double sequences $x=\left\{\xi_{k l}\right\}$ with complex-valued elements are considered. In this paper the following classes of double sequences are defined: $\mathbf{m}_{\Phi}, \mathbf{m c}_{\Phi}$, $\mathbf{r}_{\Phi}, \mathbf{l}_{\Phi}, \mathbf{m}_{\Phi}^{*}, \mathbf{m c}_{\Phi}^{*}, \mathbf{r}_{\Phi}^{*}$ and $\mathbf{l}_{\Phi}^{*}$, which generalize the well-known classes $m, m c, r$ and 1 respectively $(\S 1)$.

The author gives the method of obtaining the necessary and sufficient conditions for the linear transformations $\alpha \rightarrow \beta$, where $\alpha$ denotes one of the classes given above, and $\beta$ is one of the classes $m_{\psi}, m_{\psi}, r_{\psi}, 1_{\psi}$ or $c_{\lambda}(\$ 2)$.

Further, the necessary and sufficient condition is given for the linear transformations $\alpha \rightarrow \mathrm{I}(\alpha=\mathrm{m}, \mathrm{mc}, \mathrm{r})(\S 3)$.

Two examples are presented on the application of these conditions to the investigation of the convergence of the Cauchy product series $(\$ 4)$.

Tartu State University 